\title{
Time-resolved optical measurement of thermal transport by surface plasmon polaritons in thin metal stripes
}

\author{
A. Ganser, ${ }^{\text {a) }}$ D. Benner, R. Waitz, J. Boneberg, E. Scheer, and P. Leiderer ${ }^{\text {b) }}$ \\ Department of Physics, University of Konstanz, Universitätsstraße 10, 78464 Konstanz, Germany
}

(Received 22 September 2014; accepted 5 November 2014; published online 14 November 2014)

\begin{abstract}
We investigate the thermal transport originating from the propagation of surface plasmon polaritons (SPPs) in a thin gold stripe. The SPPs are excited by a grating coupler on the Au stripe which was patterned onto a silicon membrane. The transmissivity changes of the Si membrane due to temperature-induced changes of the interference conditions enable measuring the temperature distribution with temporal and spatial resolution better than $1 \mu \mathrm{s}$ and $1 \mu \mathrm{m}$. With this setup, we demonstrate that SPP excitation, propagation, and decay are accompanied by considerable heating and heat transport. C 2014 AIP Publishing LLC. [http://dx.doi.org/10.1063/1.4901956]
\end{abstract}

Heat management in nanoscale junctions is a fast growing field of interest in nanoscience for fundamental reasons as well as for application. ${ }^{1-13}$ In many experiments, metallic nanoelectrodes act as optical antenna for local enhancement of the electrical field created by laser irradiation. ${ }^{14,15}$ In this context, localized surface plasmons as well as running surface plasmon polaritons (SPPs) ${ }^{16-19}$ might have a major impact for the interpretation of nano-optoelectronic experiments. ${ }^{20-24}$ A prerequisite for assessing these nanoscale effects is the understanding of heat transport by plasmons in mesoscopic systems, where a local equilibrium between the electronic and the phononic system can safely be assumed and, thus, a local temperature is well-defined. In metallic nanoparticles, localized plasmons can lead to hot spots with very high field intensities, leading, e.g., to local photopolymerization of surrounding resist and even to local melting of the nanostructures. ${ }^{25,26}$ In this paper, we address propagating SPPs in micron size gold stripes and show how the lateral heat flow and dissipation by SPPs in such systems can be directly measured by optical inspection. ${ }^{27}$ Our data demonstrate that the contribution of SPPs can lead to a distinct local temperature increase, illustrating that the decay of SPPs along their propagation path has to be taken into account in the heat management of plasmonic devices.

Our experimental set-up is shown in Fig. 1(a). It relies on the temperature dependence of the optical transmissivity of a thin, free-standing $\mathrm{Si}$ membrane, as described previously. $^{27}$ The membrane (thickness $340 \mathrm{~nm}$, area $700 \mu \mathrm{m}$ $\times 700 \mu \mathrm{m})$ is homogeneously illuminated by light with a wavelength of $488 \mathrm{~nm}$ (using a high power white light light emitting diode (LED) and an optical bandpass filter $( \pm 1 \mathrm{~nm}))$, and the transmissivity is measured with a lateral resolution of about $1 \mu \mathrm{m}$ by an optical microscope combined with a sensitive CCD camera. In our measurement, a local temperature increase of $1 \mathrm{~K}$ led to a change of transmissivity of the membrane of $0.4 \%$, which was readily observable

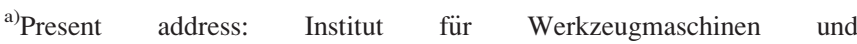
Betriebswissenschaften, TU München, Boltzmannstraße 15, 85748 Garching, Germany

b) Author to whom correspondence should be addressed. Electronic mail: Paul.Leiderer@uni-konstanz.de
}

with our set-up. By pulsing the LED, snapshots of the twodimensional temperature distribution across the membrane could be taken, with a time resolution in the range of $100 \mathrm{~ns}$ (depending on the duration of the LED probe pulse).

For the investigation of the heat release related to SPP propagation, we used samples like the one shown in Fig. 1(b). They consisted of gold stripes (width $4 \mu \mathrm{m}$, thickness $100 \mathrm{~nm}$ ), prepared on the $\mathrm{Si}$ membrane by electron beam lithography and thermal evaporation. In order to excite the SPPs, a grating was carved into the gold by focused ion beam milling, which in the measurement was then illuminated by a focused light beam (wavelength $785 \mathrm{~nm}$, spot diameter (FWHM) $3 \mu \mathrm{m}$ ) from a laser diode. The light polarization could be chosen perpendicular or parallel to the long axis of the stripe (i.e., parallel or perpendicular to the lines of the grating), allowing for a strongly suppressed or an efficient generation of SPPs, respectively. ${ }^{16,24}$ For comparison, we illuminated in some of the experiments an unstructured part of the gold stripe with the focused light of a $405 \mathrm{~nm}$ laser diode. At this wavelength, gold absorbs a major part of the incident light and the laser beam can be considered as a simple local heat source.

An example for this latter case of pure heating is presented in Fig. 2(a). The heating laser diode was periodically switched on for $2 \mu$ s (rectangular shape) at a repetition rate of $50 \mathrm{kHz}$. The illumination LED for the transmissivity measurement was synchronized with this sequence, but with a variable delay, allowing for a pump-probe measurement of the temperature distribution in the membrane. Fig. 2(a) represents a snapshot of the "temperature map" around the gold stripe at a delay time of $1.4 \mu$ s after the onset of the heating pulse. The gold stripe itself is marked black in this image. One notices a nearly isotropic (radially symmetric) temperature distribution around the heated spot. This might appear surprising at first glance, because one could have expected a pronounced asymmetry due to the contribution of the thermal transport in the gold film. Actually, comparing the thermal conductivities of $\mathrm{Si}(150 \mathrm{~W} / \mathrm{mK})^{27}$ and $\mathrm{Au}$ thin films $(173 \mathrm{~W} / \mathrm{mK})^{24,28}$ and taking into account the thicknesses of the Si membrane and the Au film, one estimates that the total heat flux in the membrane and in the gold film for a given temperature gradient is of the same order of magnitude. 

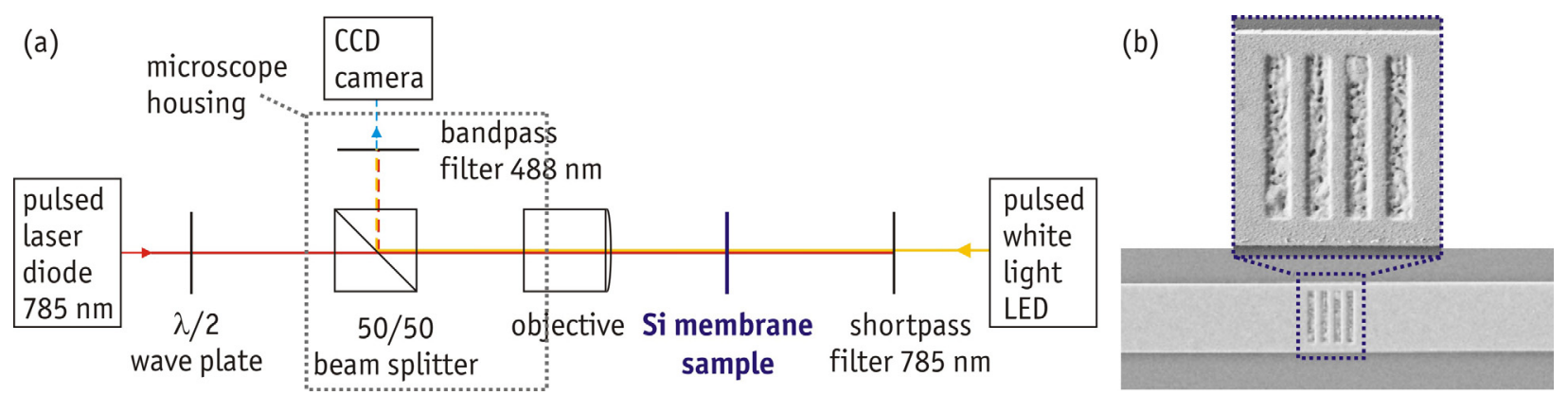

FIG. 1. (a) Optical setup. (b) SEM image of a section of a typical sample. The gold stripe (appearing bright) has a width of $4 \mu \mathrm{m}$, a total length of $300 \mu \mathrm{m}$ and a thickness of $100 \mathrm{~nm}$. The lines of the grating in the center have a period of $750 \mathrm{~nm}$. The zoom shows a closer look of the grating.

However, in our measurement, we do not determine heat fluxes, but the temperature gradients that go with them, and the relevant parameter is, hence, the thermal diffusivity $D=\kappa / c \rho$ with the thermal conductivity $\kappa$, the specific heat $\mathrm{c}$, and the density $\rho$.

Since the thermal diffusivities of the Au thin film and the Si membrane are comparable, the temperature gradient along the stripe and perpendicular to it are similar as well. This results in the observed nearly isotropic temperature distribution in Fig. 2(a).

In order to check this experimental outcome, we have also carried out simulations of the whole system, Si membrane plus Au stripe, using the COMSOL multiphysics program package. ${ }^{29}$ The heating by the laser beam was simulated by a Gaussian-shaped heat source with the same size as the experimental focus diameter. The result is depicted in Fig. 2(b) and shows that also in the calculations the distribution is fairly isotropic, corroborating the experimental observation.

We now turn to the temporal development of the temperature pattern as the heat pulse is switched on. Fig. 2(c) presents the time dependence of the temperature increase $\Delta T$ in five regions of interest (ROIs) at different distances from the heating spot, partly perpendicular to the gold stripe, and partly parallel to it, as indicated by the colored squares in Fig. 2(a). The thermal signal shows a clear response, but smeared out compared to the rectangular shape of the heating pulse, because the pulse duration is comparable to the thermal relaxation time of the system. COMSOL simulations predict that both the characteristic time for reaching a steady state temperature after switching the heating laser on and the cooling time after switching it off are in the range of a few $\mu$ s for the lateral length scales considered here, and our measurements confirm this result. One notices that for larger distances of the detection region from the laser focus, the signal has smaller amplitude and exhibits an increasing delay with respect to the heating pulse, as expected. A comparison of the traces in Fig. 2(c) for the different ROIs reveals that for the same distance from the heating spot (red and green, black and magenta), the temperature signals are similar, irrespective of the position of the ROI with respect to the gold film. This is in line with the isotropic temperature distribution seen in Fig. 2(a).

A qualitatively different behavior is observed when SPPs are excited, as shown in Fig. 3. For this purpose, a laser diode with a wavelength of $785 \mathrm{~nm}$ was used, and the light was focussed onto the grating in the gold stripe, with its polarization parallel to the long axis of the stripe. The SPPs which are generated under these conditions propagate along the gold stripe with a typical decay length of about $11 \mu \mathrm{m} .{ }^{16,24}$ This implies an additional energy transport along the gold stripe, and represents a heat source which is spread out along the stripe, as the SPPs decay into phonons. The temperature map of Fig. 3(a), which is a snapshot $1.2 \mu$ s after the onset of the laser pulse, shows now elliptically shaped isotherms with a pronounced anisotropy. This reflects the contribution of the SPPs to the energy transport in the system, which adds to the isotropic thermal transport arising from the usual optical absorption of the laser light by the grating structure.

This SPP contribution is clearly visible also in the time dependence of the temperature increase, plotted in Fig. 3(b). Two features are obvious from this graph: (i) The temperature increase close to the gold stripe (black and red) is larger
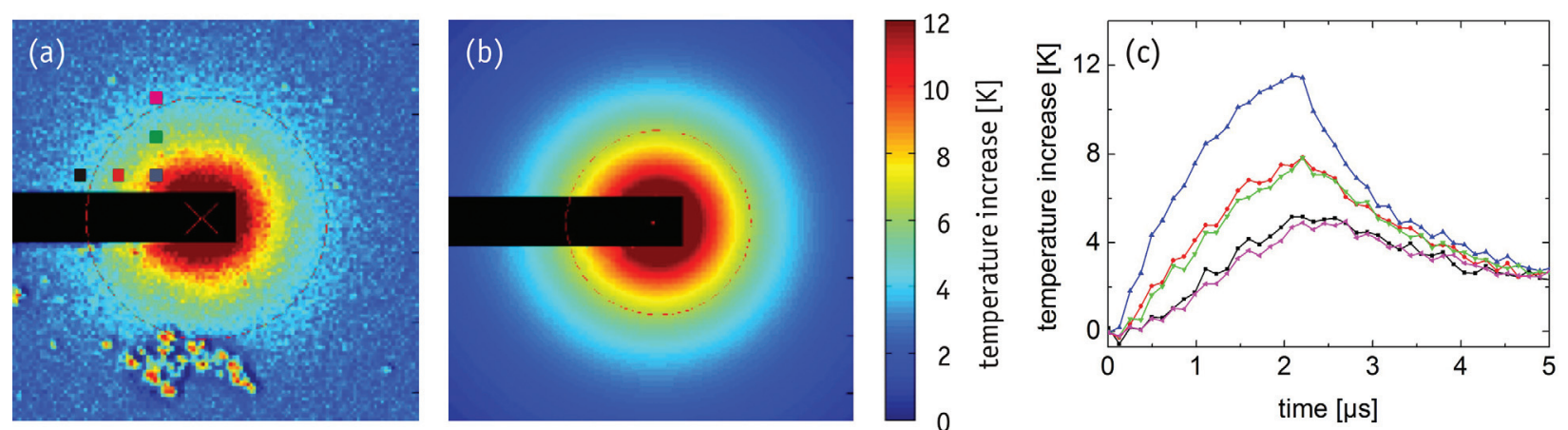

FIG. 2. (a) Experimental and (b) simulated temperature profile when irradiating an unpatterned part of the gold stripe with laser light $(\lambda=405 \mathrm{~nm})$ recorded $1.4 \mu \mathrm{s}$ after the onset of the heat pulse. The black area marks the gold stripe. The irregular dots near the bottom of Fig. 2(a) are an artefact, caused by contaminations of the Si membrane. (c) Temporal evolution of the local temperature at the positions marked by the small colored squares in Fig. 2(a). 

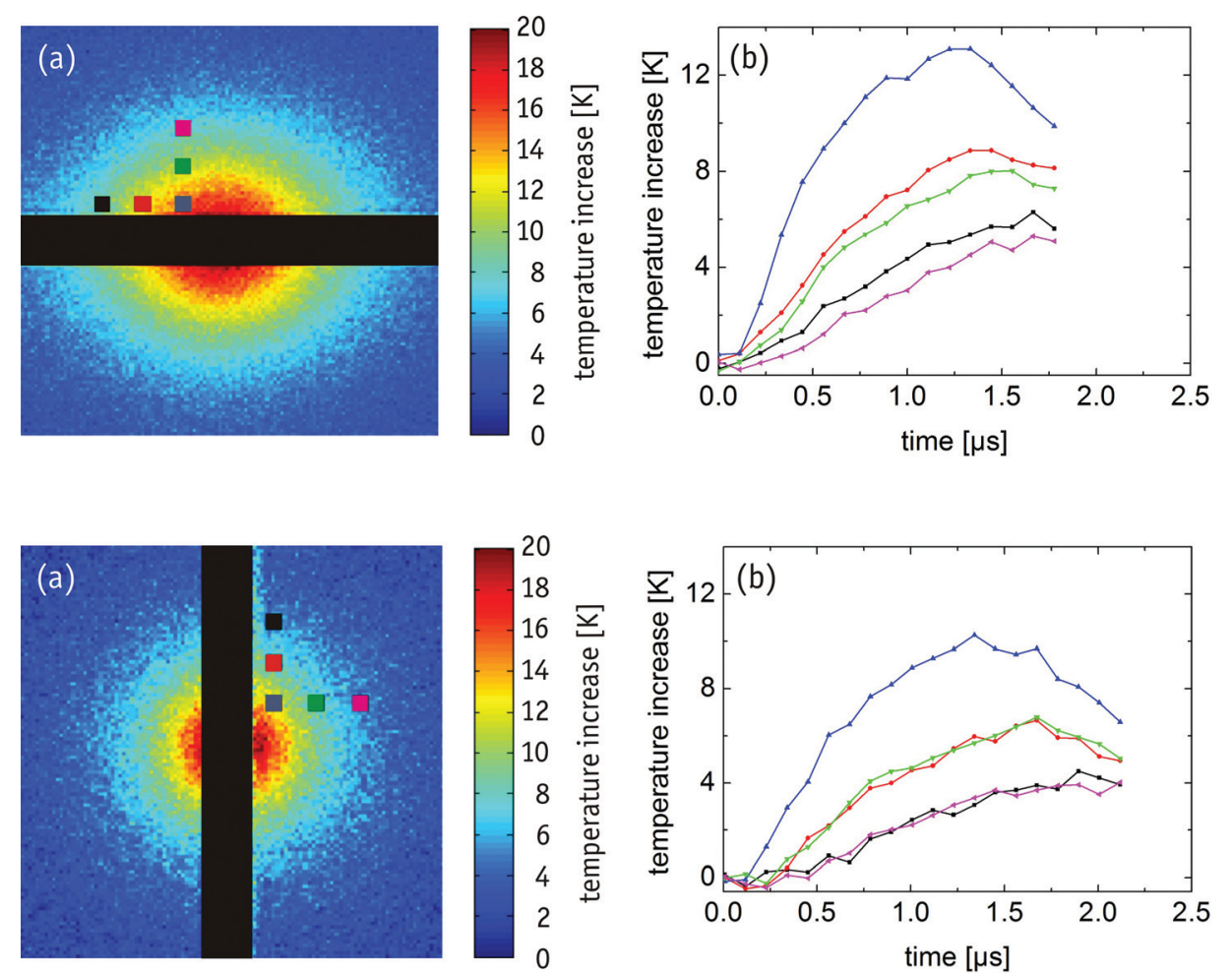

FIG. 3. (a) Experimental temperature profile when irradiating the gold stripe at the grating in the center of the image with laser light $(\lambda=785 \mathrm{~nm}, 1.2 \mu \mathrm{s}$ pulse duration and polarization along the stripe) recorded at the end of the heat pulse. The black area marks the gold stripe. (b) Temperature increase at five ROIs marked by small colored squares in Fig. 3(a).
FIG. 4. Same as Fig. 3 but for the polarization perpendicular to the gold stripe. than the one perpendicular to it (magenta and green) as already noticed in Fig. 3(a); (ii) when the laser pulse is switched on, the temperature rise at the magenta curve follows a quadratic-like behavior, which is expected for the pure thermal diffusion of phonons through the Si membrane. At the black curve, however, a linear-like contribution to the temperature increase is discernible at the beginning of the pulse, arising from heating by SPP decay. This channel provides an instantaneous heat source: the SPPs propagate along the gold stripe at a velocity of $v_{\mathrm{SPP}}=c\left(\varepsilon_{\mathrm{M}} \varepsilon_{\mathrm{D}} /\left(\varepsilon_{\mathrm{M}+} \varepsilon_{\mathrm{D}}\right)\right)$, where $c$ is the speed of light in vacuum and $\varepsilon_{\mathrm{M}}$ and $\varepsilon_{\mathrm{D}}$ are the dielectric functions of the metal (gold) and the dielectric material above the metal (air in our case). The resulting propagation velocity of SPPs is just slightly below the speed of light, very fast compared to phonon propagation. Thermal phonons resulting from SPP decay therefore arrive faster in the detection region close to the gold stripe than "normal" thermal phonons.

In a control experiment, the sample was rotated by $90^{\circ}$ with the laser still being focused on the grating. The excitation of SPPs under this condition was suppressed because the light polarization was perpendicular to the long axis of the stripe; hence, the measurement should resemble the situation in Fig. 2. This is indeed the case: As the temperature map in Fig. 4(a) illustrates, the distribution is now again nearly isotropic. Moreover, the linear temperature rise in the black curve at the beginning is missing (see Fig. 4(b)). The optical absorption of the heating laser beam by the grating, as recorded in our measurements, was about a factor of 3 smaller for the polarization in Fig. 4 as compared to the one in Fig. 3. Therefore, the power amplitude of the laser pulse was increased by the same factor in order to obtain comparable signal amplitudes for the two temperature maps.

In conclusion, we have observed an enhanced energy transport when surface plasmon polaritons (SPPs) are excited in a thin, narrow metal stripe, which is a clear signature of SPP propagation and decay. The data show that such thermal measurements can be used to gain information about SPP generation and propagation and are thus complementary to the optical detection of SPPs via light scattering or evanescent fields. ${ }^{17-19,30,31}$ Compared to previous investigations of SPPs by thermal effects, ${ }^{12,13}$ the method described here has very high spatial and temporal resolution of better than $1 \mu \mathrm{m}$ and $1 \mu \mathrm{s}$. It has therefore high potential for studying thermal phenomena in plasmonics, not only in the simple model situation addressed here, but also for imaging plasmon-mediated heating and localizing hot spots in more complex geometries, and for studies of the heat management on the nanoscale in general.

We thank H. Misawa and all the SFB discussion group members for valuable and fruitful discussions to this work. We are grateful to M. Hagner for operating the lithography and focused ion beam system and valuable advice regarding sample preparation. We also thank M. Schmotz for his contributions in the early phase of this experiment, M. Kratt for help in data processing, and S. Dickreuter for support regarding the simulations. We gratefully acknowledge financial support from the Deutsche Forschungsgemeinschaft through SFB767 and the Strategic Japanese-German Cooperative Program of the JST and DFG on Nanoelectronics, from the Ministry of Science and Arts Baden-Württemberg through the Center of Applied Photonics, and the Baden-Württemberg-Stiftung in the framework of the Research Network Functional Nanostructures.

${ }^{1}$ D. G. Cahill, P. V. Braun, G. Chen, D. R. Clarke, S. Fan, K. E. Goodson, P. Keblinski, W. P. King, G. D. Mahan, A. Majumdar, H. J. Maris, S. R. Phillpot, E. Pop, and L. Shi, Appl. Phys. Rev. 1, 011305 (2014).

${ }^{2}$ N. Li, J. Ren, L. Wang, G. Zhang, P. Hänggi, and B. Li, Rev. Mod. Phys. 84, 1045 (2012).

${ }^{3}$ Y. Dubi and M. Di Ventra, Rev. Mod. Phys. 83, 131 (2011). 
${ }^{4}$ M. J. Montgomery, T. N. Todorov, and A. P. Sutton, J. Phys.: Condens. Matter 14, 5377 (2002).

${ }^{5}$ S. Jezouin, F. D. Parmentier, A. Anthore, U. Gennser, A. Cavanna, Y. Jin, and F. Pierre, Science 342, 601 (2013).

${ }^{6}$ W. Lee, K. Kim, W. Jeong, L. A. Zotti, F. Pauly, J. C. Cuevas, and P. Reddy, Nature 498, 209 (2012).

${ }^{7}$ J. T. Lü, M. Brandbyge, and P. Hedegård, Nano Lett. 10, 1657 (2010).

${ }^{8}$ M. Galperin, K. Saito, A. V. Balatsky, and A. Nitzan, Phys. Rev. B 80, 115427 (2009).

${ }^{9}$ D. Dundas, E. J. McEniry, and T. N. Todorov, Nat. Nanotechnol. 4, 99 (2009).

${ }^{10}$ A. Pecchia, G. Romano, and A. Di Carlo, Phys. Rev. B 75, 035401 (2007).

${ }^{11}$ M. Tsutsui, S. Kurokawa, and A. Sakai, Appl. Phys. Lett. 90, 133121 (2007).

${ }^{12}$ R. W. Heeres, S. N. Dorenbos, B. Koene, G. S. Solomon, L. P. Kouwenhoven, and V. Zwiller, Nano Lett. 10, 661 (2009).

${ }^{13}$ J.-C. Weeber, K. Hassan, A. Bouhelier, G. Colas-des-Francs, J. Arocas, L. Markey, and A. Dereux, Appl. Phys. Lett. 99, 031113 (2011).

${ }^{14}$ S. Berwerger, J. M. Atkin, R. L. Olmon, and M. B. Raschke, J. Phys. Chem. Lett. 3, 945 (2012).

${ }^{15}$ C. Ropers, C. C. Neascu, T. Elsaesser, M. Albrecht, M. B. Raschke, and C. Lienau, Nano Lett. 7, 2784 (2007).

${ }^{16}$ D. Benner, J. Boneberg, P. Nürnberger, G. Ghafoori, P. Leiderer, and E. Scheer, New J. Phys. 15, 113014 (2013).

${ }^{17}$ J. R. Krenn, M. Salerno, N. Felidj, B. Lamprecht, G. Schider, A. Leitner, F. R. Aussenegg, J. C. Weeber, A. Dereux, and J. P. Goudonnet, J. Microsc. 202, 122 (2001).
${ }^{18}$ B. Lamprecht, J. R. Krenn, G. Schider, H. Ditlbacher, M. Salerno, N. Felidj, A. Leitner, F. R. Aussenegg, and J. C. Weeber, Appl. Phys. Lett. 79, 51 (2001).

${ }^{19}$ R. Zia, J. A. Schuller, and M. L. Brongersma, Phys. Rev. B 74, 165415 (2006).

${ }^{20}$ D. R. Ward, F. Hüser, F. Pauly, J. C. Cuevas, and D. Natelson, Nat. Nanotechnol. 5, 732 (2010).

${ }^{21}$ Z. Ioffe, T. Shamai, A. Ophir, G. Noy, I. Yutsis, K. Kfir, O. Cheshnovsky, and Y. Selzer, Nat. Nanotechnol. 3, 727 (2008).

${ }^{22}$ N. Ittah and Y. Selzer, Nano Lett. 11, 529 (2011).

${ }^{23}$ D. C. Guhr, D. Rettinger, J. Boneberg, A. Erbe, P. Leiderer, and E. Scheer, Phys. Rev. Lett. 99, 086801 (2007).

${ }^{24}$ D. Benner, J. Boneberg, P. Nürnberger, R. Waitz, P. Leiderer, and E. Scheer, Nano Lett. 14, 5218 (2014)

${ }^{25}$ K. Ueno, S. Juodkazis, T. Shibuya, V. Mizeikis, Y. Yokota, and H. Misawa, J. Phys. Chem. C 113, 11720 (2009).

${ }^{26}$ S. Dickreuter, J. Gleixner, A. Kolloch, J. Boneberg, E. Scheer, and P. Leiderer, Beilstein J. Nanotechnol. 4, 588 (2013).

${ }^{27}$ M. Schmotz, P. Bookjans, E. Scheer, and P. Leiderer, Rev. Sci. Instrum. 81, 114903 (2010).

${ }^{28}$ G. Langer, J. Hartmann, and M. Reichling, Rev. Sci. Instrum. 68, 1510 (1997).

${ }^{29}$ COMSOL multiphysics, version 4.3 .

${ }^{30}$ J.-C. Weeber, J. R. Krenn, A. Dereux, B. Lamprecht, Y. Lacourte, and J. P. Goudonnet, Phys. Rev. B 64, 045411 (2001).

${ }^{31}$ J.-C. Weeber, Y. Lacourte, and A. Dereux, Phys. Rev. B 68, 115401 (2003). 